\title{
SUPPORT-FREE METAL ADDITIVE MANUFACTURING: A STRUCTURED REVIEW ON THE STATE OF THE ART IN ACADEMIA AND INDUSTRY
}

\author{
Weber, Sebastian $(1,2)$; \\ Montero, Joaquin (1,2); \\ Bleckmann, Matthias (2); \\ Paetzold, Kristin (1) \\ 1: University of the Bundeswehr Munich; \\ 2: Bundeswehr Research Institute for Materials, Fuels and Lubricants (WIWeB)
}

\begin{abstract}
Especially in the Laser Powder Bed Fusion (L-PBF) technology for metals, current manufacturing systems require the use of support structure to withstand recoater forces and lower thermal induced stresses. These support structures set limitations on the design freedom and affect the surface quality, part cost and lead-time in an undesirable manner. Complex parts, which were not possible with conventional manufacturing methods, can be produced without these limitations. While some companies claim to print parts with horizontal overhangs without the use of support structure, academic research seems to deal with these limitations by defining design guidelines rather than eliminating them. In order to highlight the discrepancies between academia and industry, a structured review is presented. As result, severe differences in knowledge were discovered, which might emerge from the use of unconstrained cutting-edge systems in industry. Eventually support-free L-PBF is not yet fully developed, but the use of support structure can be drastically reduced by optimizing the build process.
\end{abstract}

Keywords: 3D printing, Additive Manufacturing, Design for Additive Manufacturing (DfAM)

\section{Contact:}

Weber, Sebastian

Bundeswehr University Munich

Department of Aerospace Engineering

Germany

s.weber@unibw.de

Cite this article: Weber, S., Montero, J., Bleckmann, M., Paetzold, K. (2021) 'Support-Free Metal Additive Manufacturing: A Structured Review on the State of the Art in Academia and Industry', in Proceedings of the International Conference on Engineering Design (ICED21), Gothenburg, Sweden, 16-20 August 2021. DOI:10.1017/ pds.2021.542 


\section{INTRODUCTION}

For a long time, additive manufacturing (AM) has been seen as the technology that enables design freedom. More than 30 years after the technology was invented, many Design for AM (DfAM) guidelines have been developed since, pointing out the limitations and opportunities of the technology. In 2019, metal materials for AM had a by $41.9 \%$ increased revenue compared to 2018 (Wohlers et al., 2020), highlighting the request for metal parts, which are frequently produced with Laser Powder Bed Fusion (L-PBF). Especially for the L-PBF with metal powder, there is currently a need for support structure and a minimum possible angle for support-free overhang structures exists. As parts are getting more and more complex to fulfil functional as well as lightweight requirements, design freedom in the way of support-free additive manufacturing is highly requested. Not only the part design would benefit from such technological improvements, but support-free printing also reduces the build time and part cost. As design processes are in motion and need to change with the evolving technology, the $45^{\circ}$ rule of thumb for overhanging structures (Protolabs, 2018) might no longer be valid. Up to the present time, there is only a small amount of experimentally-based knowledge on overhanging structures residual stress formation (Patterson et al., 2017), even though part cracking and deformation are the main reasons for build failures. In contrast to academic findings, some commercial systems claim to be capable of successfully printing parts with small or even $0^{\circ}$ overhang angles. In order to highlight the gap between academia and industry, the aim of this contribution is a review on the state of the art for support-free additive manufacturing using the metal L-PBF process.

\section{METHODOLOGY}

The scope of this contribution is to compare commercial solutions and published academic articles, which serve the overall goal of support-free printing in metal additive manufacturing. For this, a literature study was performed with Scopus and Google Scholar as literature databases. Due to the small number of available articles on the topic of support-free AM, a wide variety of keywords and acronyms was necessary for the literature search. The Design Science Research model of Hevner et al. (2004) was used as a structure for this review. The model is separated into three sections: the environment, the knowledge base and in between those two, the Design Science Research. Each main section is then further divided into sub-sections. The Design Science Research section consists of the sub-sections artefact and evaluation, where the artefact is the product of Design Science Research and the evaluation is the demonstration of the utility, quality and efficacy of a design artefact (Hevner et al., 2004). Figure 1 shows this model with the adaptations for the topic of support-free metal additive manufacturing. The relevance cycle can be seen as an information exchange, where the AM Research takes information from and gives back some kind of contribution to the environment. The contribution can be in form of new technology or machines that serve the need of the end-user. A similar exchange happens between Knowledge Base and AM research, where the deliverables are research articles or the artefact itself. The third cycle implemented in the model is the Design Cycle. It describes the iterative process of de- or refining a research artefact and the evaluation of its results. The model is described in full detail for Information Systems Research by Hevner et al. (2004).

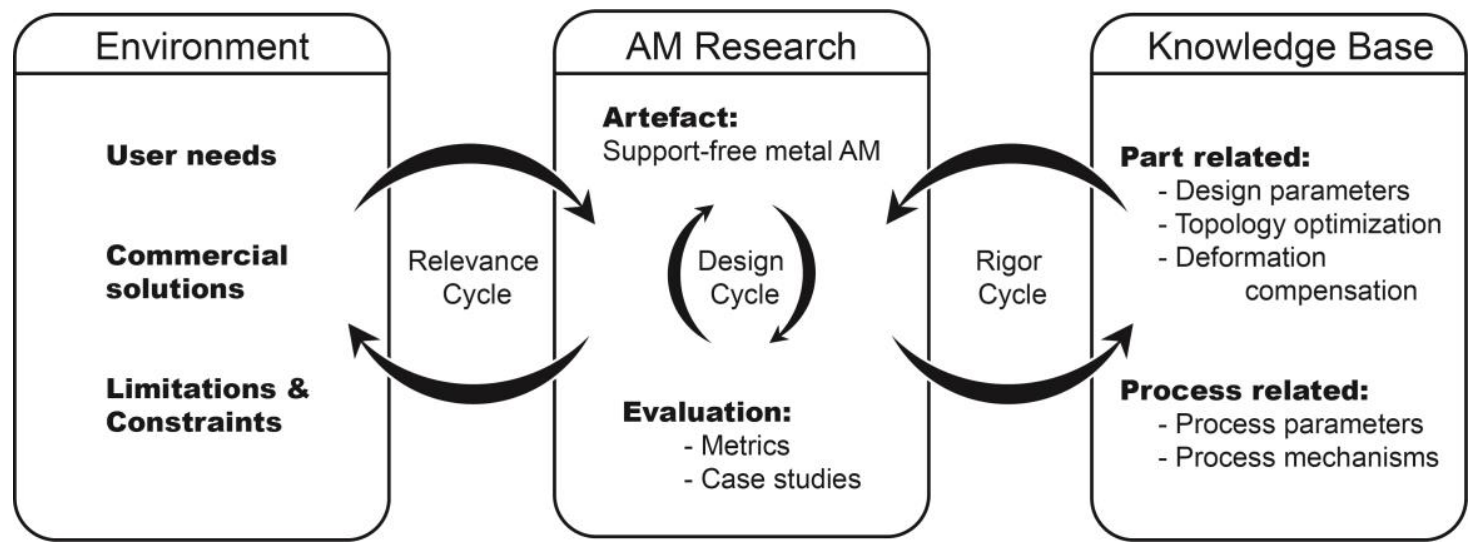

Figure 1. Design Science Research for support-free AM. Adapted from Hevner et al. (2004) 


\section{STRUCTURED REVIEW}

The following section contains the review, which is clustered in three main categories. The categories environment, AM research and knowledge base are selected following the construct of the Design Science Research model of Hevner et al. (2004) as described in the previous section. Each category includes the investigated articles but is of course not limited to those, mentioned in this work.

\subsection{Environment}

The environment category contains information about the user needs, which should be satisfied by the research or development outcomes, commercial solutions, which are such outcomes of the AM research as well as limitations and constraints that set the boundaries.

\subsubsection{User needs}

Understanding the needs of the end-user is an important aspect for the development of new technology or for conducting research. Therefore, most recent web articles were analysed. For additive manufacturing, one of the biggest needs is the increase of repeatability and consistency of the process (Boissonneault, 2019). Only in this way, successful printing on the first try can be achieved to make use of the high flexibility and the ability to easily customize parts that AM is known for (Boissonneault, 2019). The idea of using AM for functional parts comes with the urge for actual design freedom without limitations (Grimm, 2020; Valdivieso, 2020) like those, which currently apply to metal additive manufacturing processes. Without these limitations, the design freedom would allow parts to be produced that were not possible by conventional manufacturing processes in the past (Hall, 2020). Examples are any kind of internal cavities that need support structures in L-PBF processes and where the accessibility to remove those is not given or the parts aspect ratio is a limiting factor (Grimm, 2020). Further, the possibility of one-to-one transitions of parts that were not originally designed following the given DfAM guidelines would exist (Grimm, 2020). Especially on the spare part production, this would have a big impact. Producing functional parts requires a consistent surface finish (Hall, 2020), which could be achieved by reducing the hard to control manual processes included especially in postprocessing steps (Grimm, 2020; Valdivieso, 2020).

Like every manufacturing technology, also AM profits from reduced cost and labour. The current $60 \%$ to $70 \%$ of parts needing CNC machining after the build process (Grimm, 2020) show the existing potential and need for reducing the post-processing effort (Boissonneault, 2019). With the reduced necessity for post-processing, lead-times are also getting shorter. Another reason for this long lead-time in AM is the iterative re-design process, which is necessary to make parts suitable for the manufacturing technology (Boissonneault, 2019). The part cost can be further reduced by the stacking of parts in the build chamber to achieve larger batch sizes and use the process more efficiently (Valdivieso, 2020).

\subsubsection{Commercial solutions}

Currently, there are different Laser Powder Bed Fusion systems for metals available on the market. Each of these systems comes with a specific configuration of the energy source and the recoating system. These from each other differing configurations lead to variations in the capability of printing overhang structures. The machine manufacturer 3D Systems for example uses a proprietary recoating system, which makes it possible to print overhang angles down to $20^{\circ}$ relative to the base plate using the machine DMP 100 Flex. Together with the reduced necessity for support structures compared to competing companies, this leads to a reduced post-processing effort and an increased surface quality (3D Systems, 2018).

One of their competitors is the machine manufacturer Velo3D, who claims that the Sapphire system is a support-free metal additive manufacturing system, because of the possibility to print unsupported $0^{\circ}$ overhang angles and high aspect ratios of up to 3000:1 for various, including the titanium alloy Ti6Al4V (Velo3D, 2020). Nevertheless, also Velo3D has limitations with printability, which is for example a maximum span of $10 \mathrm{~mm}$ for $0^{\circ}$ overhang bridges (Miller, 2020). Velo3D achieves these results by targeting the software, focussing on closed-loop control as well as the development of their heavily patented recoater system (cf. Buller et al., 2016), which utilizes vacuum for bed levelling and a noncontacting recoating approach instead of traditional blade scraping. Closed-loop control is understood by Velo3D as focussing on quality management by continuously monitoring and correcting print parameters 
during the process, while their idea for software is to minimize the many steps that are necessary between part design and manufacturing (Boissonneault, 2019).

Another party that is trying to overcome the issue of printing small overhang angles is the German company EOS. Recently they published test reports showing a homogeneous downside surface of parts printed with a $10^{\circ}$ overhang angle on their EOS M290 metal L-PBF machine using 316L stainless steel and a hard recoater blade (Wohlfart, 2020). Other tests conducted by EOS include the manufacture of $100 \mathrm{~mm}$ discs of the titanium alloy Ti6Al4V also on the M290 with a hard recoater blade. The outline of the discs was supported by a $1 \mathrm{~mm}$ contour support leaving the inside as unsupported $0^{\circ}$ overhang. The scan strategy was adapted that no contour scans and no scan pattern were involved. Additionally, the exposure strategy had gradually changing parameters over the build height to adapt the energy input to the loose powder situation. Parameter tests were carried out on 36 cylinders, which are displayed in Figure $2 \mathrm{~b}$ ) having multiple discs at different heights with a $60 \mu \mathrm{m}$ segmentation for layers to apply the parameters, which were optimized for each layer. As can be seen in Figure 2 a), the resulting parts showed a slightly increased surface roughness, which can be compared to the roughness of a normal overhang structure. (Wohlfart, 2019a)

Further tests reported by EOS were conducted on an EOS M290 machine using Ti6A14V titanium powder. The aim of these tests was part stacking as shown in Figure $2 \mathrm{c}$ ) without support fixation in the build chamber. As the parts still need to be supported against the recoater forces, a supporting shell with a $0.2 \mathrm{~mm}$ clearance between the shell and the part is used. The friction of the powder, which is remaining in the gap, is used for support, while still allowing easy part removal by hand. The test sample, which is shown in Figure 2 d) is a self-supporting upside-down tree with lattice structure created in nTopology and a solid trunk for stacking. (Wohlfart, 2019b)

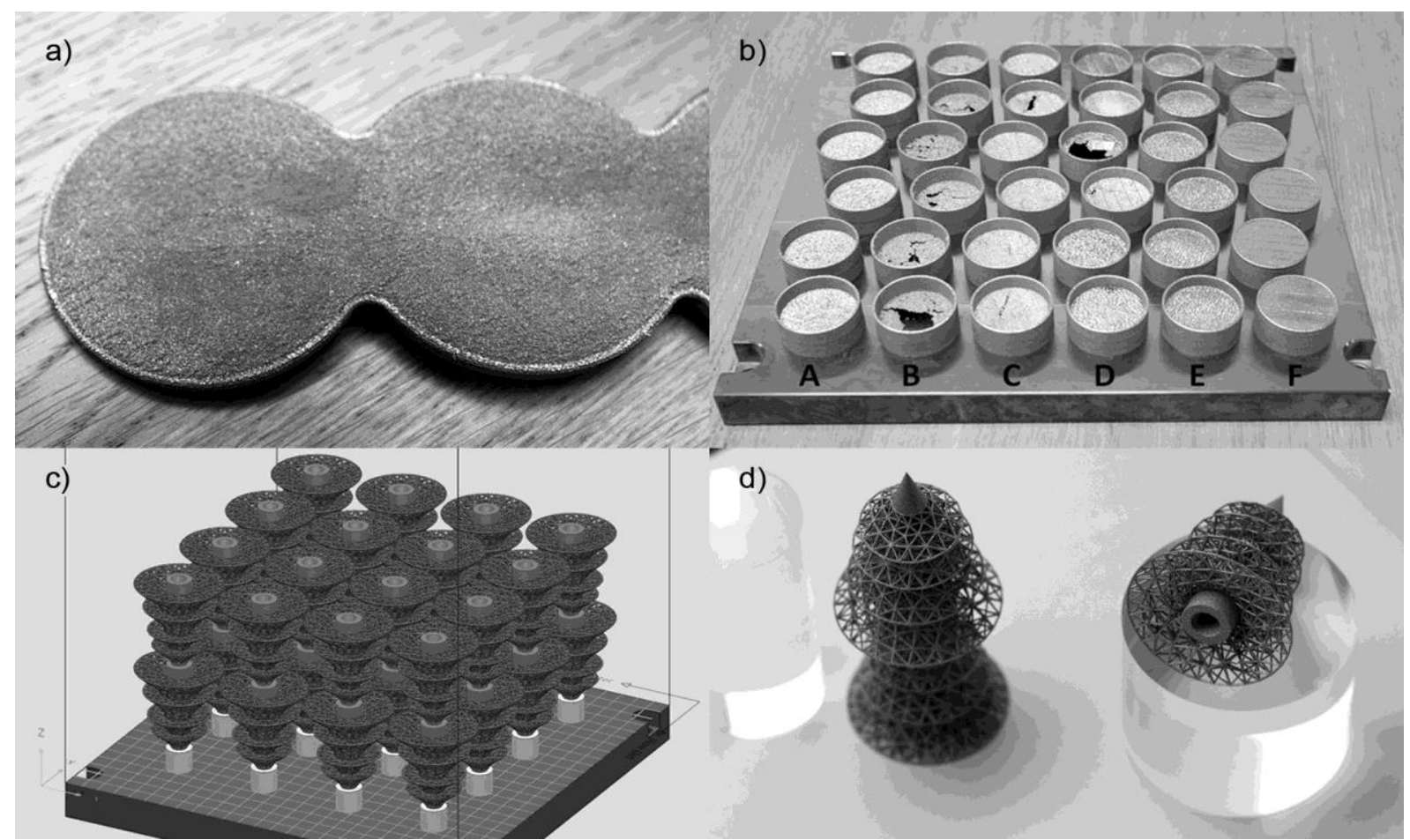

Figure 2. Down skin surface of discs in a) with corresponding parameter tests in b). Part stacking during build preparation in c) and printed tree in d). Pictures courtesy of EOS GmbH.

\subsubsection{Limitations}

Limitations can be either temporarily or consistent over time. Patents belong to the first group and hinder the development of new technology because they restrict the adaption of existing technology. Nevertheless, patents are granted for a specified period, which allows the use and adaption after their expiration. Due to the broad descriptions in the legal writing style of patents and the therefore imprecise explanation of patented systems, patents are not included in this review. Laws of physics on the other hand belong to the second group and set boundaries to the possible solutions. Dealing with these boundaries by different technological approaches might counteract their effect, but cannot obliterate the laws themselves. For example, the use of support structure is a way to circumvent the issue of not being 
able to print metal parts directly into the loose powder, while accepting the drawback of support removal with additional post-processing steps. Additional physical limitations can be the residual stress formation or overheating due to bad thermal conductivity.

\subsection{Knowledge Base}

For this literature study, the Knowledge Base is seen as the part, to which mostly academic research contributes. It sets the foundation for further investigations. For the topic of AM research, the Knowledge Base was divided into part related items and process related items, which are presented in the following subsections.

\subsubsection{Part related}

The part related items are further structured into the topics of design parameters, topology optimization and deformation compensation, while the focus for this contribution should still remain in the design aspect. The design parameters group contains research items that are related to DfAM rules and guidelines with a spotlight on parameters for overhang structures.

\section{Design parameters:}

Thompson et al. (2016) give generic information about design opportunities, considerations and constraints. They state that design guidelines that handle process specific limitations are given by the machine manufacturer Stratasys and the software company Materialise. Furthermore, the authors suggest choosing specific processes, which suit the required material and way of manufacturing, or to modify the geometry to circumvent the limitations of a given process. Only a small section of their work is about support structures and overhangs. They mention that even for self-supporting geometries the thermalinduced residual stresses sometimes require support structures to counteract these effects. As a conclusion, they derived that part orientation, support structure, process and machine parameters are considered a single entity and cannot be developed independently.

The AM service provider Protolabs (2018) also provides guidelines for the use of support and the handling of overhanging geometry. The company defines overhangs as abrupt changes in geometry, which are called a bridge if the overhang is supported by two or more features. In its general guidelines and rules of thumb for metal additive manufacturing, the company suggests a self-supporting angle of $45^{\circ}$ and a maximum overhang distance of $0.5 \mathrm{~mm}$ for $0^{\circ}$ overhangs. According to them, bridges should be designed with a maximum span of $2 \mathrm{~mm}$ and holes with a maximum diameter of $8 \mathrm{~mm}$.

A set of guidelines, derived from physical testing on an EOSINT M270 Xtended and a ConceptLaser M2 L-PBF machine with Ti6Al4V titanium powder, was given by Kranz et al. (2015). They see the necessity of such rules, because of the many unwanted design iterations that are necessary for a successful print. The conducted tests include the determination of critical overhang angles for build failure. Their investigations showed an increased powder adhesion and thereby bad surface quality for angles lower than $40^{\circ}$ and the need for support with angles smaller than $10^{\circ}$ from the horizontal. They conclude that given those parameters, the printability is still highly dependent on the machine, the recoating angle as well as the used material.

In contrast to complete design guidelines, Atzeni and Salmi (2015) focus only on self-supporting overhangs using default parameters on an EOSINT M270 Xtended and the aluminium alloy AlSi10Mg. They investigated flat, convex and concave shapes for overhang angles between $35^{\circ}$ and $45^{\circ}$. Their results showed that concave geometry is more suitable for large overhang angles, while small angles benefit from concave structures.

Instead of investigating the limits of what can and cannot be done, Cloots et al. (2013) presented a strategy to tackle the post-processing effort and manufacturing costs by reducing the number of necessary supports. The strategy is the segmentation of the geometry in critical and uncritical regions, where supports are needed only for $0^{\circ}$ overhangs. This was possible by a reduced energy input for overhang layers and so-called connection layers above support structures with process parameters that differ from the standard parameters. The strategy was tested on a ConceptLaser M2 with 316L stainless steel. Results showed that a minimum of 20 connection layers is required and that part stacking is possible with the use of minimal support structure.

Recently, Zhang et al. (2020) investigated unsupported bridge geometries with simulations and experiments using AlSi10Mg powder. They unveiled that bridge structures can restore their designed profile after a certain amount of layers, whilst lever structures tend to fail, because of the missing support 
on the second side. Nevertheless, the thickness at the centre point of the bridge is reduced due to preceded warping of the down face. They also report a beneficial effect of reduced energy input on the formation of the upward bend.

A completely different strategy was proposed by Cooper et al. (2017). Even though it was presented for an electron beam manufacturing technology, they claim that their idea can be also applied to L-PBF. The proposed strategy involves so-called heat support, which uses non-contacting support as a heat sink to reduce the thermal-induced residual stresses. A gap of nine layers was used between the part and the support structure. The concept proofed to be suitable only for small overhangs, as larger unsupported geometries needed additional fixation due to an increased thermal-stress induced moment.

\section{Topology optimization:}

As topology optimization is not primarily the topic of this review, only a few examples that are directly related to the reduction of the support structure are given. One publication to be mentioned is the one from Li et al. (2017), in which topology optimization is used to find lightweight structures fulfilling support-free requirements, such as a critical inclination angle, minimum and maximum diameters as well as a maximum overhang distance. Their approach requires manual inspection by experienced designers for evaluation of printability and subsequent re-design to achieve a final support-free design. They were able to reduce unnecessary weight with a consecutive size optimization, but had still $20.8 \%$ increased volume compared to the original optimization result.

While the previously presented approach used additional material to satisfy the support-free requirements, Wang et al (2018) had the idea of locally adapting the geometry instead of adding material, to stick closer to the original optimization result. They utilized a level-set based algorithm with the signed distance property as a constraint in form of a domain integral. In contrast to conventional approaches, this constraint is able to detect down facing non-self-supporting points. The presented topology optimization is carried out after the optimal orientation of the part is found.

Lastly, Langelaar (2018) combined geometry, support layout and build orientation in a simultaneous optimization instead of inefficient constraint inhibited topology optimizations. He proposed the use of support cost as an optimization criterion, which is based on support volume and a cost factor. The resulting designs using his approach show only a single contact to the base plate, which leads to a weak heat transfer and thus an unstable process.

\section{Deformation compensation:}

A short insight will also be given into deformation compensation, keeping in mind that compensating means counteracting the effect instead of tackling the cause. The work of Paggi et al. (2019) should be mentioned, as it describes the investigation of down-facing surfaces with overhang angles between $25^{\circ}$ and $75^{\circ}$. The results showed that unsupported edges tend to bend upwards due to residual stresses and the deformation can be compensated by applying correction factors, which are developed within the study.

The idea of deformation compensation is taken one step further by Hong et al. (2020). They trained a neural network with point cloud data to perform the compensation. Their driving factor is that process optimization strategies are not capable of fully eliminating geometrical imperfections while existing compensation approaches also have trouble with highly irregular shapes. The developed approach was tested with steel on an EOS M290, proofing the successful print of overhang angles down to $30^{\circ}$.

\subsubsection{Process related}

The second subsection of the Knowledge Base contains process related items, which are further structured into process parameters and mechanisms. Items are added to the parameter topic if they tackle specific build parameter sets, whereas mechanism items help to better understand the build process.

\section{Process parameters:}

Starting in 2013, Wang et al. (2013) performed initial studies on the effect of energy density on overhang angles. For their tests, they used 316L stainless steel on a self-developed machine with $200 \mathrm{~mm} / \mathrm{s}$ and $600 \mathrm{~mm} / \mathrm{s}$ at $150 \mathrm{~W}$ followed by tests between $200 \mathrm{~mm} / \mathrm{s}$ and $1200 \mathrm{~mm} / \mathrm{s}$ for different laser power and angles between $25^{\circ}$ and $50^{\circ}$. Their initial calculated minimum possible angle of $27^{\circ}$ showed to be true, while in general, increasing laser speed led to better results and less warping. They concluded that with higher energy input, the probability of failure increases. This result was confirmed by a simulative and 
experimental study on 316L steel melt tracks for overhanging geometries at $40^{\circ}$ angle using an SLM 500HL performed by Le et al. (2020). They additionally observed that high laser power together with low energy input leads to less discontinuity.

Another study on energy input was presented by Chen et al. (2017). They simulated thermal behaviour of AlSi10Mg powder to find the optimal energy density for best fabricating quality. Their results showed that the perfect range for energy is between $60 \mathrm{~J} / \mathrm{mm}^{3}$ and $80 \mathrm{~J} / \mathrm{mm}^{3}$ with best results for small layer thicknesses and high scanning speeds, qualitatively supporting the results of Wang et al. (2013).

DePond et al. (2018) also investigated the variation of laser power, but for a $316 \mathrm{~L}$ steel geometry with a $6 \mathrm{~mm}$ wide internal channel, similar to a bridge structure with a 2,5 and 10 layer overhang. The laser power varied between $40 \mathrm{~W}$ and $382 \mathrm{~W}$ at $400 \mathrm{~mm} / \mathrm{s}$ scanning speed. They concluded that specific overhang region parameters lead to lack of fusion if applied to the whole layer.

A similar insight was used in the work of Mertens et al. (2014), where the scan strategy was adapted for down-facing structures using 5 zones. Parameter tests conducted on a self-developed machine with AlSi10Mg lead to $60 \mathrm{~W}$ for zone 1 instead of $300 \mathrm{~W}$ regular power, which would lead to unstable and large melt pools on loose powder, because of the low heat conductivity. The developed strategy uses a minimum of 10 layers per zone and gradually increasing laser power for zones 2 to 5 .

The scan strategy was also subject of the work of Bagg et al. (2016), which focuses on scan patterns for arches built with the alloy 718. Distortions after cut-off and residual stress in build direction were affected in the same way that they were the largest for a chess pattern, followed by an island pattern, showing the smallest values for a continuous strategy. Residual stresses in $\mathrm{x}$-direction were affected in reversed order with the smallest values for continuous scan paths and the largest for the chess pattern.

Cloots et al. (2017) also investigated adapted process parameters with a shell and core exposure strategy. Using their strategy, they were able to print unsupported overhangs down to $30^{\circ}$ on a ConceptLaser M2 and a Renishaw AM250 using 316L steel with best results for a thick shell. They suggest starting the shell scans from the inside to assure the best heat conductivity.

\section{Process mechanisms:}

To further understand the process Chivel (2013) monitored the melt pool temperature and penetration into $3 \mathrm{~mm}$ of loose powder. Investigated materials were copper, cobalt-chromium and 316 $\mathrm{L}$ steel. He determined that the gravity field leads to a Rayleigh-Taylor instability between the melt pool and the loose powder. Hussein et al. (2013) also investigated 316L steel directly in the powder bed. They used finite element simulations to understand the connection between induced heat and residual stresses. Their results revealed that reduced cooling rates and shorter scanning tracks reduce the possibility of cracks. Another simulative study on 316L steel single tracks in loose powder was performed by Mohanty and Hattel (2016) with the aim of reliability characterization. Their contribution is an increased prediction accuracy of simulations by reducing the uncertainty given by physical parameters.

While the previous publications indirectly affected the creation of overhangs, Zhang et al. (2018) investigated the effect of the recoater angle. Tests with Ti6AL4V on an EOSINT M280 showed that for small angles the warpage in recoater direction is suppressed, while it is encouraged against the recoater direction. Within their study, they were able to reduce the number of necessary supports by $35 \%$ on average by orienting the part dependent on the recoating direction.

A completely different approach for support-free printing is proposed by Vora et al. (2015). Their anchorless SLM (L-PBF) utilizes a pre-heating up to $380^{\circ} \mathrm{C}$ for successfully printing $5 \mathrm{~mm}$ and $10 \mathrm{~mm}$ overhangs with a maximum of $1 \mathrm{~mm}$ deformation. They used AlSi12 on an adapted Renishaw 125 for an unsupported T-shaped geometry. Conclusions were that pre-heating leads only to the desired effect if insitu alloying is used since normal powder tends to agglomerate during the process.

\subsection{AM Research}

The AM research is the part that includes the design cycle as a continuous process of refining the artefact and evaluating the results. The artefact, which should benefit from this review, is the not yet accomplished research goal and therefore only case studies are presented as additional literature for evaluation of the current state.

\subsubsection{Artefacts}

As this literature review only uses the described Design Science Research model as structure instead of applying it, the artefact is the topic of the review and not a research item. Meaning that the aim of 
support-free printing is considered the artefact that should be accomplished by the AM community, consisting of academic researchers as well as $R \& D$ engineers in industry. The artefact was shaped over time in several design cycles starting from full exploitation of the AM potential by applying DfAM guidelines towards setting new boundaries in support minimization, finally ending up with support-free printing.

\subsubsection{Evaluation}

In order to evaluate the current state of AM research and whether the artefact is complete or not, specific metrics need to be selected. These metrics allow the evaluation of discrete values, which can be determined using case studies. An example of such metrics for the artefact of support-free printing can be the post-processing time, which is needed to remove existing supports. Another metric would be the minimum printable angle without the process to fail. This metric can be applied to a case study that was presented by the company Delva, showing the successful print of a stator ring with several $2 \mathrm{~mm}$ thick blades. These blades with overhang angles down to $13^{\circ}$ were printed without the use of support structure. The stator ring was printed on an EOS machine with a hard recoater blade and optimized process parameters as well as an adapted exposure strategy. As the stator ring was a spare part and re-design was not possible, support-free printing was necessary. (Nimelä, 2020)

A featured article by the company Renishaw gives three exemplary case studies, which involve none or a minimum amount of supports. The first part is a seat post for a bike, which was topology optimized with a $45^{\circ}$ overhang constraint. In this way, the resulting part needs only support to be anchored to the base plate. The second and third case studies present designs, which violate the DfAM guidelines for overhanging structures, but cannot be re-designed to completely avoid support structure. In both cases, the support structure is specifically designed to fit the required application. (Saunders, 2017)

\section{DISCUSSION \& CONCLUSION}

An extensive literature review was performed, which has the aim to highlight on the one hand side the gap between academia and industry and on the other hand reveals the state of the art. The review showed that process parameters and their effects on process mechanisms are not yet fully understood and are highly dependent on the build environment. Not only the machines and the material but also the part design as well as the volumetric proportion between part and powder play an important role in the printability of overhanging structures. Most of the presented studies used 316L stainless steel powder as build material, which exhibits less residual stresses as the in aerospace and the medical field commonly used titanium alloy Ti6Al4V and is therefore more forgiving in terms of printability. Available knowledge must be distinguished between support-free printing, which is achieved by following DfAM guidelines and printability that is achieved by unveiling a higher level of design freedom based on adapted technology. Plenty of research was conducted for the development of DfAM guidelines and to better understand the process mechanisms, which can be applied to existing machines. In general, academic research seems trying to deal with existing challenges or limitations rather than finding and eliminating their cause, while industry seems to achieve this objective.

Severe differences in knowledge between industry and the academic field were discovered. Some of the discrepancies might result from the use of newly developed machinery in industry, which may not yet be available on the market or the fact that companies develop and patent new ways of manufacturing without contributing to the Knowledge Base. Another reason might be that commercial manufacturing systems, which are used for studies in academia, give no full control over build parameters. As an example, it is not possible to change the energy input and scan strategy during the build based on continuous process monitoring, evaluation and adaption.

The review showed that the general known $45^{\circ}$ angle for support-free printing could be reduced to $30^{\circ}$, as several studies showed resulting angles down to or less than $30^{\circ}$ using different machines and materials with optimized process parameters. The tests conducted by EOS showed that even for the difficult to print titanium powder, unsupported flat overhangs can be achieved on a regular L-PBF machine with rather good surface quality, once the process parameters and scan strategy are adapted to the loose powder.

As conclusion can be stated that the artefact of support-free AM is not yet fully developed. Future research should try to further understand the relationship between material properties and overhang 
printability. In addition, new L-PBF in-process methods need to be developed, which aim to reduce the recoater forces and in-process part deformation in order to reach the full support-free capabilities.

\section{CONFLICT OF INTEREST}

The selected brands and products are presented for information purpose only. The authors are not affiliated with any of these companies. Therefore, the authors declare no conflicting interests.

\section{REFERENCES}

3D Systems. (2018), "DMP Flex 100”, 3D Systems, available at: https://www.3dsystems.com/3d-printers/dmpflex-100 (accessed 2 November 2020).

Atzeni, E. and Salmi, A. (2015), "Study on unsupported overhangs of AlSi10Mg parts processed by Direct Metal Laser Sintering (DMLS)", Journal of Manufacturing Processes, Vol. 20, pp. 500-506.

Bagg, S.D., Sochalski-Kolbus, L.M. and Bunn, J.R. (2016), "The Effect of Laser Scan Strategy on Distortion and Residual Stresses of Arches made with Selective Laser Melting", NASA Technical Reports, presented at the American Society of Precision Engineering (ASPE) 2016 Summer Topical Meeting: Dimensional Accuracy and Surface Finish in Additive Manufacturing, Huntsville.

Boissonneault, T. (2019), "VELO3D: breaking barriers in metal AM with support-free 3D printing”, 3D Printing Media Network, 3 July, available at: https://www.3dprintingmedia.network/velo3d-metal-am-support-freeinterview/ (accessed 2 November 2020).

Buller, B., Milshtein, E. and Seelinger, S. (2016), “Apparatuses, systems and methods for three-dimensional printing. U.S. Patent No. 9,346,127 B2”, Santa Clara, CA, 24 May.

Chen, H., Gu, D., Xiong, J. and Xia, M. (2017), "Improving additive manufacturing processability of hard-toprocess overhanging structure by selective laser melting”, Journal of Materials Processing Technology, Vol. 250, pp. 99-108.

Chivel, Y. (2013), "Investigations of the selective laser melting of the overhang layers", in Veiko, V.P. and Vartanyan, T.A. (Eds.), , presented at the Fundamentals of Laser Assisted Micro- and Nanotechnologies 2013, St. Petersburg, Russian Federation, available at:https://doi.org/10.1117/12.2052541.

Cloots, M., Spierings, A. and Wegener, K. (2013), “Assessing new support minimizing strategies for the additive manufacturing technology SLM”, Solid Freeform Fabrication Symposium (SFF), Austin, TX, pp. 12-14.

Cloots, M., Zumofen, L., Spierings, A.B., Kirchheim, A. and Wegener, K. (2017), “Approaches to minimize overhang angles of SLM parts”, Rapid Prototyping Journal, Vol. 23 No. 2, pp. 362-369.

Cooper, K., Steele, P., Cheng, B. and Chou, K. (2017), "Contact-Free Support Structures for Part Overhangs in Powder-Bed Metal Additive Manufacturing”, Inventions, Vol. 3 No. 1, available at:https://doi.org/10.3390/inventions3010002.

DePond, P.J., Guss, G., Ly, S., Calta, N.P., Deane, D., Khairallah, S. and Matthews, M.J. (2018), “In situ measurements of layer roughness during laser powder bed fusion additive manufacturing using low coherence scanning interferometry", Materials \& Design, Elsevier, Vol. 154, pp. 347-359.

Grimm, T. (2020), "The Business Impact of a Support-Less Process for Metal Additive Manufacturing", Campbell, CA, 30 January.

Hall, H. (2020), "Support structures: the need, impact, and elimination strategies", R\&Dworld, 31 January, available at: https://www.rdworldonline.com/support-structures-the-need-impact-and-elimination-strategies/ (accessed 2 November 2020).

Hevner, A.R., March, S.T., Park, J. and Ram, S. (2004), "Design science in information systems research”, MIS Quarterly, JSTOR, pp. 75-105.

Hong, R., Zhang, L., Lifton, J., Daynes, S., Wei, J., Feih, S. and Lu, W.F. (2020), “Artificial neural network-based geometry compensation to improve the printing accuracy of selective laser melting fabricated sub-millimetre overhang trusses", Additive Manufacturing, available at:https://doi.org/10.1016/j.addma.2020.101594.

Hussein, A., Hao, L., Yan, C. and Everson, R. (2013), "Finite element simulation of the temperature and stress fields in single layers built without-support in selective laser melting”, Materials \& Design (1980-2015), Vol. 52, pp. 638-647.

Kranz, J., Herzog, D. and Emmelmann, C. (2015), "Design guidelines for laser additive manufacturing of lightweight structures in TiAl6V4", Journal of Laser Applications, Vol. 27 No. S1, available at:https://doi.org/10.2351/1.4885235.

Langelaar, M. (2018), "Combined optimization of part topology, support structure layout and build orientation for additive manufacturing”, Structural and Multidisciplinary Optimization, Vol. 57 No. 5, available at:https://doi.org/10.1007/s00158-017-1877-z.

Le, K.Q., Wong, C.H., Chua, K.H.G., Tang, C. and Du, H. (2020), "Discontinuity of overhanging melt track in selective laser melting process", International Journal of Heat and Mass Transfer, Vol. 162, available at:https://doi.org/10.1016/j.ijheatmasstransfer.2020.120284. 
Li, Z., Zhang, D.Z., Dong, P. and Kucukkoc, I. (2017), “A lightweight and support-free design method for selective laser melting”, The International Journal of Advanced Manufacturing Technology, Vol. 90 No. 9-12, pp. 2943-2953.

Mertens, R., Clijsters, S., Kempen, K. and Kruth, J.-P. (2014), “Optimization of Scan Strategies in Selective Laser Melting of Aluminum Parts With Downfacing Areas”, Journal of Manufacturing Science and Engineering, Vol. 136 No. 6, available at:https://doi.org/10.1115/1.4028620.

Miller, G. (2020), “How to Build the Better Fuel Tank, Fast!”, presented at the VeloVirtual Series, 4 November.

Mohanty, S. and Hattel, J.H. (2016), "Improving accuracy of overhanging structures for selective laser melting through reliability characterization of single track formation on thick powder beds", in Gu, B., Helvajian, H. and Piqué, A. (Eds.), , presented at the SPIE LASE, San Francisco, California, United States, available at:https://doi.org/10.1117/12.2212621.

Nimelä, V. (2020), “Together we exceed any limitations of 3D printing - Delva”, Delva - 3D-Tulosteita Kovaan Käyttöön, 10 November, available at: https://delva.fi/en/news/together-we-exceed-any-limitations-of-3dprinting/ (accessed 17 November 2020).

Paggi, U., Sinico, M., Thijs, L., Dewulf, W. and van Hooreweder, B. (2019), "Improving the dimensional accuracy of downfacing surfaces of additively manufactured parts", Proceedings of the Special Interest Group Meeting on Advancing Precision in Additive Manufacturing, presented at the 2019 euspen and ASPE Special Interest Group Meeting: Advancing Precision in Additive Manufacturing, pp. 35-38.

Patterson, A.E., Messimer, S.L. and Farrington, P.A. (2017), "Overhanging Features and the SLM/DMLS Residual Stresses Problem: Review and Future Research Need”, Technologies, Vol. 5 No. 2, p. 15.

Protolabs. (2018), "How to Design and Manufacture Metal 3D-Printed Parts", Protolabs, available at: https://www.protolabs.com/resources/design-tips/how-to-design-and-manufacture-metal-3d-printed-parts/ (accessed 2 November 2020).

Saunders, M. (2017), “Can you build AM parts without supports?”, available at: https://www.renishaw.com/en/canyou-build-am-parts-without-supports--43421 (accessed 2 November 2020).

Thompson, M.K., Moroni, G., Vaneker, T., Fadel, G., Campbell, R.I., Gibson, I., Bernard, A., et al. (2016), "Design for Additive Manufacturing: Trends, opportunities, considerations, and constraints", CIRP Annals, Vol. 65 No. 2, pp. 737-760.

Valdivieso, C. (2020), "What are the business benefits of support-less metal additive manufacturing?", 3Dnatives, 26 February, available at: https://www.3dnatives.com/en/support-less-metal-additive-manufacturing260220204/ (accessed 2 November 2020).

Velo3D. (2020), “Sapphire Printer - Product Brief”, October, available at: https://www.velo3d.com/wpcontent/uploads/2020/10/PB-Sapphire-v2_0-weightier.pdf (accessed 17 November 2020).

Vora, P., Mumtaz, K., Todd, I. and Hopkinson, N. (2015), "AlSi12 in-situ alloy formation and residual stress reduction using anchorless selective laser melting", Additive Manufacturing, Vol. 7, pp. 12-19.

Wang, D., Yang, Y., Yi, Z. and Su, X. (2013), "Research on the fabricating quality optimization of the overhanging surface in SLM process", The International Journal of Advanced Manufacturing Technology, Vol. 65 No. 9-12, pp. 1471-1484.

Wang, Y., Gao, J. and Kang, Z. (2018), "Level set-based topology optimization with overhang constraint: Towards support-free additive manufacturing”, Computer Methods in Applied Mechanics and Engineering, Vol. 339, pp. 591-614.

Wohlers, T., Campbell, R.I., Diegel, O., Huff, R. and Kowen, J. (2020), Wohlers Report 2020 3D Printing and Additive Manufacturing State of the Industry.

Wohlfart, M. (2019a), “Can you Build a 100 mm Support-free Horizontal Disk?”, Linkedin, 11 March, available at: https://www.linkedin.com/pulse/can-you-build-100-mm-support-free-horizontal-disk-michael-wohlfart/ (accessed 2 November 2020).

Wohlfart, M. (2019b), "Building without support? Possibilities and limitations", Linkedin, 12 November, available at: https://www.linkedin.com/pulse/building-without-support-possibilities-limitations-michael-wohlfart/ (accessed 2 November 2020).

Wohlfart, M. (2020), “ $10^{\circ}$ unsupported overhang in 316L with a hard recoater?”, Linkedin, 28 September, available at: https://www.linkedin.com/posts/michael-wohlfart_eos-m290-teamwork-activity-6727155259044569088KYwj (accessed 2 November 2020).

Zhang, K., Fu, G., Zhang, P., Ma, Z., Mao, Z. and Zhang, D.Z. (2018), "Study on the Geometric Design of Supports for Overhanging Structures Fabricated by Selective Laser Melting”, Materials, Vol. 12 No. 1, available at:https://doi.org/10.3390/ma12010027.

Zhang, X., Wang, J., Kang, J., Rong, Y., Duan, G., Wu, P. and Zheng, L. (2020), "The dynamic arch bending mechanism of flat bridge structure of AlSi10Mg during SLM process", Materials \& Design, Vol. 188, available at:https://doi.org/10.1016/j.matdes.2020.108469. 